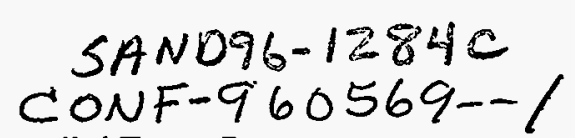

Paper for: 12th Int. Conf. on Plagma Surface Interactions in Controlled Fusion Devices - DRAFr

\title{
Characterization of Energetic Deuterium Striking the Divertor of the DIII-D Tokamak
}

\author{
R. Bastasz, W. R. Wampler, and J. A. Whaley \\ Sandia National Laboratories, U.S.A. \\ D. G. Whyte \\ INRS-Energie et Materiaux, Canada \\ P. B. Parks, N. H. Brooks, W. P. West, and C. P. C. Wong \\ General Atomics, U.S.A.
}

\begin{abstract}
Measurements of the deuterium particle flux and energy to the divertor of the DIII-D tokamak during a series of plasmas that terminated in disruptions have been made using a silicon collector probe installed on the divertor materials exposure system (DiMES). During the steady state portion of each discharge, the probe was located under the separatrix, but immediately before disrupting the plasma, by injecting either $\mathrm{Ar}$ or $\mathrm{D}_{2}$ gas, the strike point of the outer divertor leg was positioned over the probe. Comparison of the amount of retained $D$ in the probe for the two types of disruptions indicates that much of the trapped $D$ could have resulted from exposure in the private llux zone prior to the disruption. Measurements of the depth distribution of the trapped D in the Si imply that the incident ion energy was approximately $100 \mathrm{eV}$ at normal incidence and decreased slightly at oblique angles. The measurements give an upper bound to the energy of deuterons striking the divertor floor in the vicinity of the strikepoint during disruptions.
\end{abstract}

\section{Introduction}

The flux and energy of particles striking the divertor during steady state operation and during disruptions are parameters of central interest in the design of power producing tokamaks. The energetic particle flux to the divertor is a critical factor, as it has a large effect on material behavior and the lifetime of plasma-facing components [1]. To characterize the properties of plasma particles striking the divertor during plasma operations in the DIII-D tokamak, an experiment was conducted using the divertor materials exposure system (DiMES) to trap particles during exposure to plasmas that terminated in a disruption. The experiment was designed to collect the fast charge- 


\section{DISCLAIMER}

Portions of this document may be illegible in electronic image products. Images are produced from the best available original document. 
exchange (CX) D neutrals emitted from the recycled cold neutral layer, which served as a CX target for the incident ions. After exposure, the collector assembly was removed and examined using two ion beam analysis methods to obtain information about the fux and energy of the incident deuterons. Analysis of the experiment suggests that much of the trapped deuterium originated from exposure in the private flux zone prior to the disruptions and provides an upper bound to the energy of particles striking the divertor floor during a disruption.

\section{Experimental Method}

The assembly to collect energetic particles emitted from the plasma consisted of a collector, a slotted aperture plate, and a graphite cover. The collector was a polished disk of high-purity Si, which is efficient at trapping implanted atoms of the hydrogen izotopes [2]. A slotted plate, containing $1 \mathrm{~mm}$ wide slots in stainless steel, was positioned on top of the collector and provided four view angles, $\beta$, into the divertor plasma at $0^{\circ}, 30^{\circ}, 45^{\circ}$, and $60^{\circ}$ from the normal. The graphite cover protected the assembly and presented a smooth graphite surface flush with the divertor floor. Fig. 1 shows the collector assembly arrangement. Collimation by the cover and slotted plate allowed only neutral particles traveling in straight trajectories across the toroidal magnetic field to strike the Si sample.

The DiMES mechanism was used to control the pasition of the collector assembly [3]. During conditioning of the vacuum vessel the assembly was retracted beneath the divertor loor. After conditioning and a series of test plasmas to set up the appropriate plasma parameters, it was inserted into position on the divertor floor for exposure to a series of plasma disruptions. The plasma's outer strike point (OSP) is positioned outboard of the DiMES radial location, so the collector resides in the private flux region during startup and during the steady state portion of the discharge. Data were collected for two types of disruptions occurring in DIII-D: radiative disruptions induced by a large Ax gas puff, and a density limit dismuption triggered by enhanced $\mathrm{D}_{2}$ injection. One Si collector was exposed to three consecutive Ar disrupted discharges and another to a single density limit diszuption. A fast-scanning IR camera indicated that the peak heat flux to the divertor floor during the thermal quench of the Ar disruption was $\approx 300 \mathrm{MW} / \mathrm{m}^{2}$ for $2 \mathrm{~ms}$. This occurred about $2 \mathrm{~cm}$ outside of the radial location of the collector assembly, where the heat flux averaged $\approx 70 \mathrm{MW} / \mathrm{m}^{2}$. In the case of the density limit disruption, the peak heat flux to the floor was $<100 \mathrm{MW} / \mathrm{m}^{2}$ and the "footprint" was $>5 \mathrm{~cm}$ outboard of the collector. No significant heat flux hit the DiMES location.

The X-point was located directly above the assembly during formation of the plasma, so the collector was sheltered in the private flux zone of the plasma. For the Ar induced disruptions, the outer strike point (OSP) of the ohmic plasma was swept to the DiMES radius at $1.5 \mathrm{~s}$ of the plasma discharge. Beams were injected at $1.7 \mathrm{sec}$ with the number of sources being rising in stepwise fashion from one to five in 200 msec. A strong puff of Ar at $2.0 \mathrm{~s}$ initiated the disruption. The programmed location of the OSP was moved slightly further inward with each successive shot, because it was observed that the disruption caused the OSP to jump outward. It should be noted that the major heat load and particle flux in the discharges with disruptions triggered by Ar was detected at the inner strike point. 
After exposure, the collector was removed for external analysis. The amount of $D$ retained in the $S i$ was measured by nuclear reaction analysis (NRA) using the $D\left({ }^{3} \mathrm{He}, \mathrm{p}\right)^{1}$ He reaction. The depth distribution of the trapped $\mathrm{D}$ was measured at each view angle using low-energy direct recoil spectroscopy (DRS). For DRS, a $1.00 \mathrm{keV} \mathrm{Ne}{ }^{f}$ beam was used for the analysis and to erode the sample surface. An energy analyzer, positioned at an observation angle of $25^{\circ}$ with respect to the incident beam direction, recorded the intensity of positive ions emitted near the elastic recoil energy of $D(0.27$ $\mathrm{keV})$. For each profile, the signal was integrat,ed for a constant incremental $\mathrm{Ne}^{+}$dose, which was monitored by measuring the ion current on the sample. The sputter erosion rate was determined by measuring the final crater depths with a profilometer.

Depth profiles were generated by converting the dose scale to depth, and the recoil signal to $D$ concentration. The depth conversion was made using an erosion rate derived from the profilometry measurements. The concentration scale was assigned by first integrating the background subtracted signal and then scaling the normalized signal at each depth by the total amount of trapped D, determined by NRA, divided by the depth increment.

\section{Results}

The main results are given in Figs. 2 and 3, which show the amounts of retained $D$ in each sample and the depth profile of the trapped D for the Ar disruption sample at each view angle. Replicate profiles were recorded and are included to indicate the reproducibility of the depth analysis. The profiles for the $0^{\circ}$ and $30^{\circ}$ view angles show definite subsurface peak concentrations of $D$ while the $D$ is nearer the surface in the $45^{\circ}$ and $60^{\circ}$ profiles. The saturation level of $\mathrm{D}$ in bulk $\mathrm{Si}$ is about 0.5 atomic fraction, which is equivalent to $25 \mathrm{D} / \mathrm{nm}^{3}$ [4]. Beneath the surface, this value was not exceeded. At the surface, higher equivalent concentrations were observed at the oblique view angles.

Comparing the measured depth of the $\mathrm{D}$ in the Si probe with the energy dependence of the mean projected range gives an indication of the incident particle energy. A range curve has been calculated by Brice for D in Si based on Magee's experimental measurements [5]. Assuxning a monoenergetic incident flux, the indicated energy for D striking the sample is $100 \pm 50 \mathrm{eV}$ at the normal view angle. The incident particle energy appears to be slightly lower at the other view angles. No data are available on the penetration depth of the $\mathrm{D}$ for the density limit disruption exposure.

Given the incident energy, the $D$ reflection coefficient can be estimated. The relevant values were obtained by interpolating from calculations reported by Eckstein [6]. These values indicate that the trapping efficiency at $60^{\circ}$ is about a factor of two less than it is at normal incidence. The inferred incident fluences (areal density $/\left(1-R_{N}\right)$ ) average about $3 \times 10^{16} \mathrm{D} / \mathrm{cm}^{2}$. As shown in Fig. 2, there does not appear to be a strong angular dependence.

It is worth noting that the particle energy and fluence implied by the measurements indicates that substrate sputtering during the exposure was negligible, assuming that $D$ is the only species striking the sample. The sputter yield of $100 \mathrm{eV} D$ on $S^{i}$ is about $10^{-2}$ at normal incidence [7] and should not rise by more than a factor of two in going to $60^{\circ}$. The maximum amount of Si erosion by $\mathrm{D}$ bombardment that could have resulted under these conditions is not more than 0.3 of a monolayer. 
To help understand the infiuence of particle energy and view angle on the depth profiles, a number of implant distributions were calculated using the TRIM code $[8,9]$. The calculations were restricted to energies $\geq 100 \mathrm{eV}$, a region where the interatomic potcntial is considered reliable. In general, as the energy decreases the profiles narrow, move closer to the surface, and a higher fraction of the incident flux becomes implanted at the peak of the distribution. In going from normal to glancing angles of incidence at a given particle energy, the distributions again move toward the surface, but with smaller fractions of the incident fiux being implanted due to the increasing reflection coefficient. At low incident energies ( $<300 \mathrm{eV}$ ), the peak depth does not scale like cos $\beta$, as it does at higher energies [10].

Comparing the trends in the calculated and measured profile supports the inference that the incident particle energy was lower at the more glancing view angles. The experimental profiles narrow and move to the surface more sharply than can be attributed to simply the variation in the angle of incidence. Because the $\mathrm{D}$ range is so shallow, it is difficult to give an exact value for the incident energy at the $45^{\circ}$ and $60^{\circ}$ view angles. It appears to be below $90 \mathrm{eV}$.

TRIM calculations show that normally implanted $100 \mathrm{eV} \mathrm{D}$ comes to rest within $10 \mathrm{~nm}$ of the surface. The experimental profile at $0^{\circ}$ indicates that some $D$ penetrated deeper than $10 \mathrm{~nm}$ into the Si. This suggests that the incident particle energy spectrum was not strictly monoenergetic, but had a higher energy component, such as would occur in a more Maxwelliar-like distribution.

\section{Discussion}

A previous report by Parks et al. discussed the interpretation of the Ar disruption results [11]. A model was described which predicted the neutral flux collected by the slots due to CX neutrals emanating from a cold neutral layer which formed at the divertor floor (and hence directly on top of the collector) during the disruption. The model was based on the idea that ions with different pitch angles with respect to the magnetic field line have different path lengths through the neutral layer and suffer different amounts of $C X$ attenuation. Fence, the variation in the size of the neutral flux admitted by different slot angles can provide information on the ion pitch angle distribution and the thickness of the neutral layer. This analysis assumed that the measured neutral flux originated solely from the disruption event and showed that a reasonably large portion (up to $30 \%$ ) of the incident ions would have to be attenuated due to CX events in order to explain the measurements. However, the model predicted a substantially lower flux at the $0^{\circ}$ slot relative to the canted slots, which was not secrl in the experimental results. The density limit disruption experiment measured D areal densities which were larger than for the Ar induced distuptions, even though the probe was not directly hit by the disruption footprint and that it was exposed to only a single discharge. These facts raise concerns as to whether the measured $\mathrm{CX}$ flux came primarily from the disruption events.

The collected CX flux from the steady-state plasma portion of the exposure prior to the disruption can be estimated from measurca divertor plasma parameters. The private flux region of the divertor is a region of enhanced recycling and has a relatively high neutral pressure (typically $P_{\mathrm{p} i}=1 \mathrm{~m}$ Torr $=P_{\text {rnidplane }} \times 100$ for DIII-D). This region is sharply bounded by the divertor plasma, which has typical measured parameters of 
Papcr for: 12th Int. Conf. on Plasma Surface Interactions in Controlled Fusion Devices - DRAFT

$n_{c}=5 \times 10^{13} \mathrm{~cm}^{-3}, T_{e}=50-100 \mathrm{eV}$ at the separatrix with cross-field scale lengths on the order of $1 \mathrm{~cm}$ on the private flux side of the separatrix. The volumetric CX rate is determined by the product of the plasma's ion density $\left(n_{i} \approx n_{e}\right)$, the neutral density $\left\langle n_{0} \approx 3.5 \times 10^{16} P_{\mathrm{pf}}\right)$ and the CX rate coefficient $\left(\langle\sigma v\rangle_{\mathrm{Cx}} \approx 4 \times 10^{-8} \mathrm{~cm}^{3} / \mathrm{s}\right.$ at 100 $\mathrm{eV})$. There exists a thin layer of interaction between the neutral gas and the plasma where CX can take place, with the thickness of this layer being determined primarily by the electron impact ionization mean free path of the neutrals into the plasma ( $\left.l_{c x}=v_{t h} / n_{e}(\sigma v\rangle_{\text {ion }} \geq 0.1 \mathrm{~cm}\right)$. The expected CX flux, $\Gamma_{c x}$, measured at the collector is then given by

$$
\Gamma_{c x}=n_{i} n_{0}\langle\sigma v\rangle_{c x} l_{c \infty}(\Omega / 4 \pi) \text {. }
$$

where $\Omega$ is the solid angle of the collecting slot (e.g. $\Omega \approx 0.1$ sr for the $0^{\circ}$ slot). Using the typical values stated above, one calculates $\Gamma_{c x} \approx 3 \times 10^{16} \mathrm{~s}^{-1} \mathrm{~cm}^{-2}$. The total private flux exposure time for both experiments was close to one second, therefore one irnrnediately notes that the expected areal density of $3 \times 10^{16} \mathrm{D} / \mathrm{cm}^{2}$ (i.e., $300 \mathrm{D} / \mathrm{nm}^{2}$ ) matches the experimental results in Figure 3. Also, the measured ion energy of $100 \mathrm{eV}$ would be expected from the $X$-point region of the divertor plasma. One expects sorne CX flux into all the slots from this process. The $0^{\circ}$ slot will collect CX particles from the $\mathrm{X}$-point, while the other angles view progressively further down the divertor leg, toroidally displaced from the position of DiMES.

These arguments lead us to conclude that the collected CX deuterium may have resulted from both the disruption and the steady-state private flux exposure. It is not prescntly possible to discern which condition contributes the greater flux. However, the results do place an upper limit on the energy of the ions near the strike point during the disruption of $<300 \mathrm{eV}$, since no appreciable population of $\mathrm{D}$ is seen past 20 nm depth. Experiments to study plasmas without disruption events are needed and being planned. Nevertheless, the collector is successful as a diagnostic for measuring the CX flux and energy to the divertor floor. The diagnostic is expected to be useful in studying ion temperature distributions and CX power losses during detached divertor plasma conditions, as well as further studies of disruptions.

\section{Acknowledgment}

This work was supported by the US Department of Energy under contracts DEAC04-94AL85000and DE-AC03-89ER51114.

\section{References}

[1] J. Brooks, et al., these Proceedings.

[2] S. A. Cohen and G. M. McCracken, J. Nucl. Mater. 84 (1979) 157.

[3] C. P. C. Wong, et al., Procoedings of the 1996 American Nuclear Society Annual Meeting (in press).

[4] B. L. Doyle, W. R. Wampler, D. K. Brice, and S. T. Picraux, J. Nucl. Mater. 93-4 (1980) 551.

[5] C. W. Magee, S. A. Cohen, D. E. Voss, and D. K. Brice, Nucl. Instrum. Methods $\underline{168}(1980) 383$. 
[6] W. Eckstein, "Calculated Trapping Curves of D in C and Si," Max-PlanckInstitute Report IPP 9/33 (1980) $8 \mathrm{pp}+22$ figs.

[7] H. H. Andersen and H. L. Bay, in: "Sputtering by Particle Bombardment I," R. Behrisch, Ed., (Springer-Verlag, Berlin, 1981) pp 145-218.

[8] J. P. Biersack and L. G. Haggmark, Nucl. Instrum. Methods 174 (1980) 257.

[9] J. F. Ziegler, J. P. Biersack, and U. Littmark, "The Stopping and Range of Ions in Solids, Volume 1," (Pergamon, New York, 1985) 321 pp.

[10] W. Eckstein, "Computer Simulation of Ion-Solid Interactions," (Springer-Verlag, Berlin, 1991) pp 136-138.

[11] P. B. Parks, et al., Proceedings of the 1995 IEEE Symposium on Fusion Enginucering, (in press).

\section{DISCLAIMER}

This report was prepared as an account of work sponsored by an agency of the United States Government. Neither the United States Government nor any agency thereof, nor any of their employees, makes any warranty, express or implied, or assumes any legal liability or responsibility for the accuracy, completeness, or usefulness of any information, apparatus, product, or process disclosed, or represents that its use would not infringe privately owned rights. Reference herein to any specific commercial product, process, or service by trade name, trademark, manufacturer, or otherwise does not necessarily constitute or imply its endorsement, recom. mendation, or favoring by the United States Government or any agency thereof. The views and opinions of authors expressed herein do not necessarily state or reflect those of the United States Government or any agency thereof. 


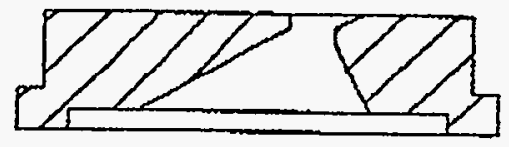

cover
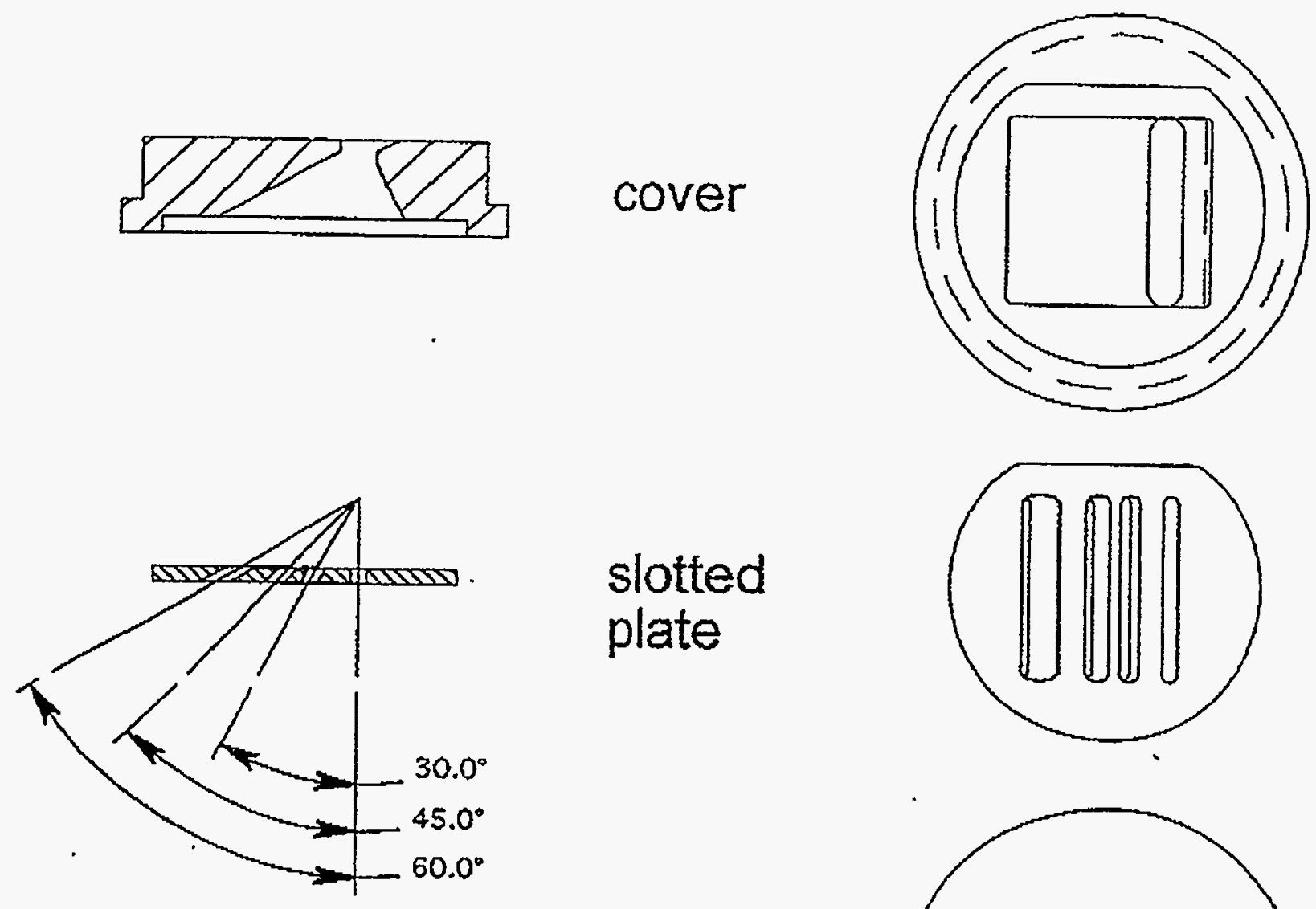

slotted

plate
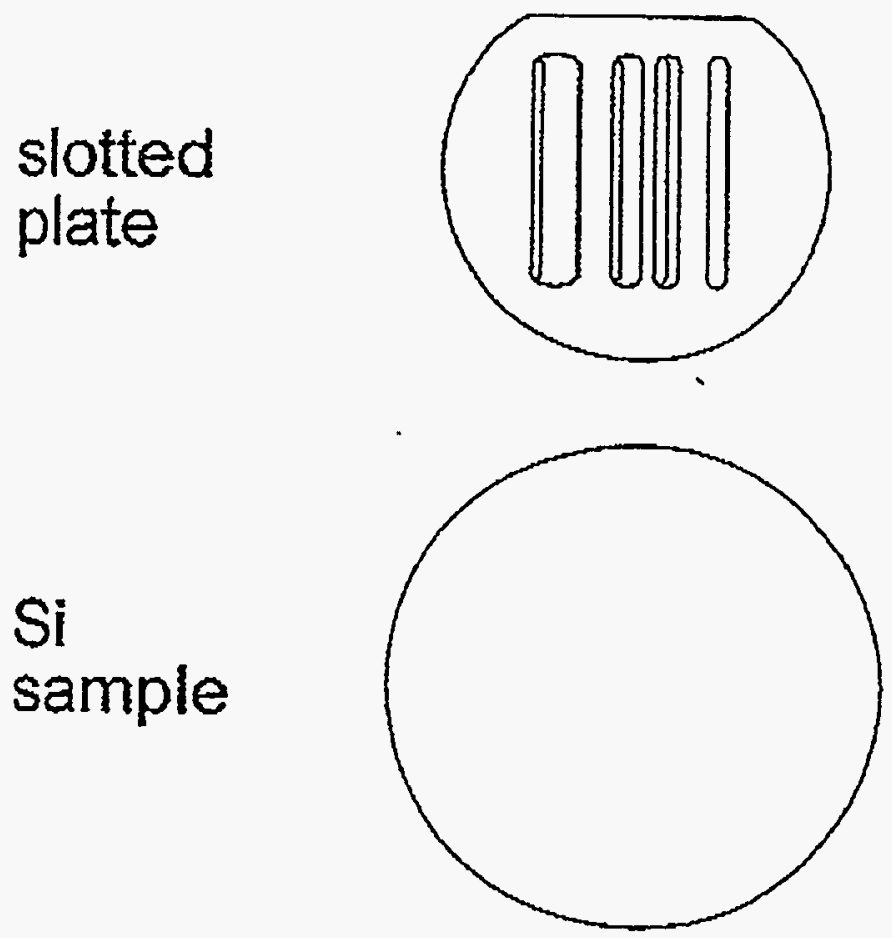

Figure 1. Diagram of the DiMES sample holder assembly used for the disruption experiment. The collector sample was a $2.54 \mathrm{crn}$ disk of high purity Si. The slotied plate (stainless steel) and cover (graphite) provided four collimated views toward the plasma flow direction. The assembly permitted only neutral particles to pass through to the sample surface. 


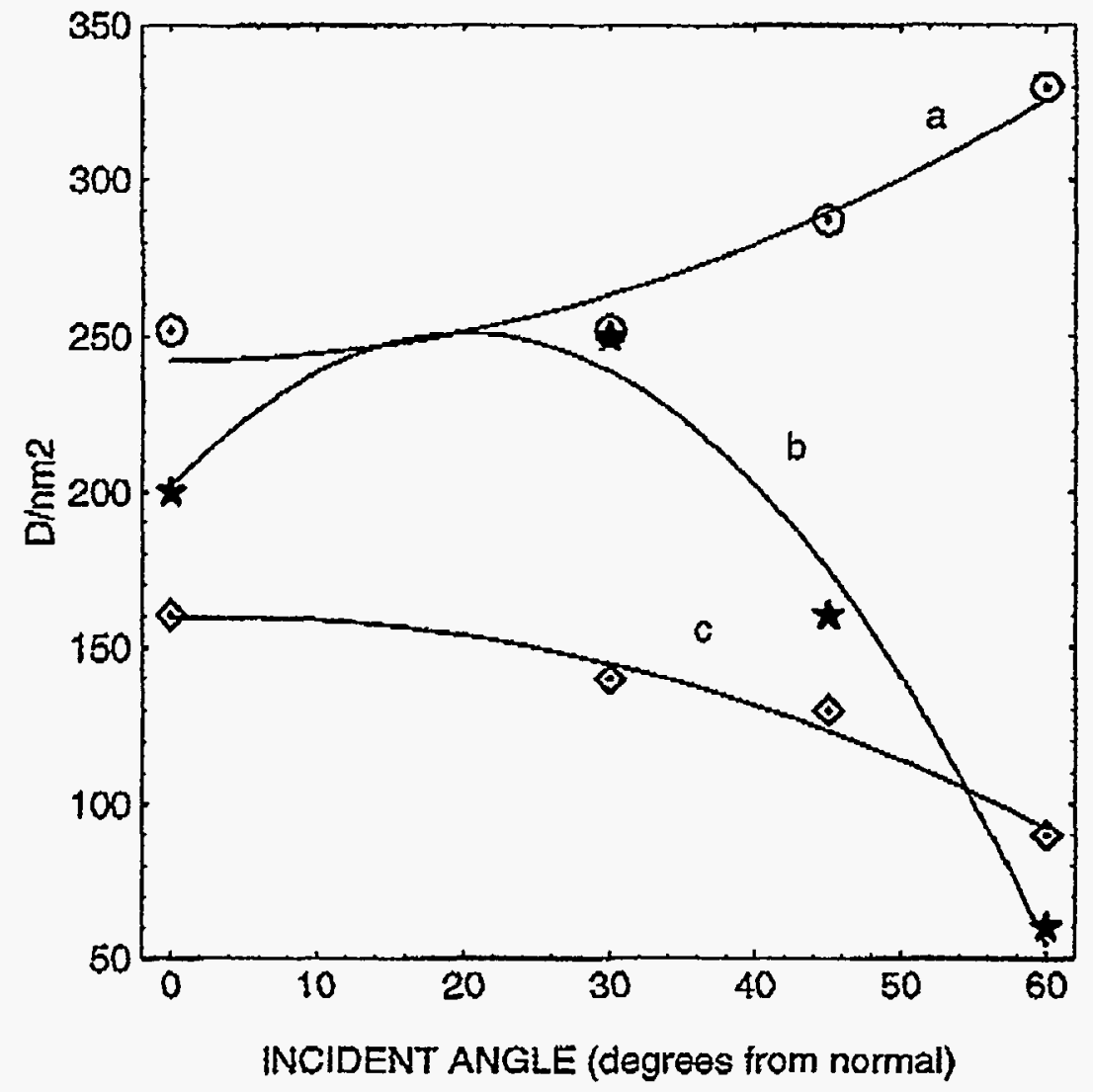

Figure 2. Measured deuterium retention (points) in the collector samples exposed to DIII-D plasmas with $A r$ and $D_{2}$ initiated disruptions. Also shown is the inferred fluence to the sample exposed to plasmas with Ar induced disruptions. The curves are fits to the data. (a) Fluence to Ar disruption sample, (b) Areal density of $\mathrm{D}$ in density limit disruption sample, (c) Areal density of $D$ in Ar disruption sample. 